\title{
Steven Johnson Syndrome: Adverse Drug Reaction
}

\section{Chandaluri $\mathbf{P}^{*}$, Prabhanjan M}

Pharm D, Bangalore University Bangalore, India

"Corresponding author: Chandaluri P, Pharm D, Bangalore University, Bangalore, India, Tel: 9966103661; E-mail: praggu23@gmail.com

Received date: January 15, 2018; Accepted date: January 18, 2018; Published date: January 22, 2018

Copyright: (c) 2018 Chandaluri P, et al. This is an open-access article distributed under the terms of the Creative Commons Attribution License, which permits unrestricted use, distribution, and reproduction in any medium, provided the original author and source are credited.

\begin{abstract}
The Steven-Johnson Syndrome (SJS) is a rare immune complex mediated hypersensitivity condition. Here we report the case of Steven-Johnson Syndrome due to the co-administration of aceclofenac and cefixime. NSAIDs are the rare type of medication that causes SJS and cefixime is a traditional medication that causes the condition. A 22years-old patient was admitted in the hospital with a complaint of fever for 15 days and he was treated by giving aceclofenac, cefixime and general symptomatic treatment. On the 4th day of treatment erythromatous patches were observed throughout the body. Laboratory investigations were performed and the case was identified as StevenJohnson Syndrome. The treatment was withdrawn and he was administered with steroids, amoxicillin, metronidazole and general symptomatic treatment. The patient has recovered after a week.
\end{abstract}

Keywords: Steven-Johnson syndrome; Ulcers; Cefixime

\section{Introduction}

Stevens-Johnson syndrome (SJS) and toxic epidermal necrolysis (TEN) are two forms of a life-threatening skin condition, in which cell death causes the epidermis to separate from the dermis. The syndrome is thought to be a hypersensitivity complex that affects the skin and the mucous membranes. The majority of cases are idiopathic (without a known cause). The main known cause is certain medications, followed by infections and rarely cancers [1].

Stevens-Johnson syndrome (SJS) usually begins with fever, sore throat, and fatigue, which is misdiagnosed and usually treated with antibiotics. Ulcers and other lesions begin to appear in the mucous membranes, almost always in the mouth and lips but also in the genital and anal regions. Those in the mouth are usually extremely painful and reduce the patient's ability to eat or drink. Conjunctivitis of the eyes occurs in about $30 \%$ of children who develop SJS. A rash of round lesions about an inch across arises on the face, trunk, arms and legs, and soles of the feet, but usually not the scalp [2].

Medications that have traditionally been known to lead to SJS, erythema multiform and toxic epidermal necrolysis include sulphonamides (antibiotic), penicillin (antibiotic), cefixime (antibiotic), barbiturates (sedative), lomotrigine and phenytoin (eg.dilantin) (anticonvulsants). Combining lamotrigine with sodium valproate increases the risk of SJS. Non-steroidal anti-inflammatory drugs are a rare cause of SJS in adults; the risk is higher for older patients, women and those initiating treatment. Typically, the symptoms of drug-induced SJS arise within a week of starting the medication. People with systemic lupus erythematosus or HIVinfections are more susceptible to drug-induced SJS [3].

Cefixime is an oral third generation cephalosporin antibiotic used to treat infections caused by bacteria. These include infections of the: Ear, Nose, Sinuses, Throat, Chest, Lungs, Urinary system and uncomplicated gonorrhoea caused by Neisseria gonorrhoeae. Adverse Drug Reactions include diarrhoea, dyspepsia, nausea, vomiting. Hypersensitivity reactions like skin rashes, utricaria and Stevens-
Johnson syndrome. Aceclofenac is an NSAID; NSAIDs are usually indicated for the treatment of acute or chronic conditions where pain and inflammation are present. Adverse drug reaction include gastrointestinal (GI) and renal effects. NSAIDs are also implicated in some cases of Stevens-Johnson syndrome.

\section{Objectives}

To report the AE's and to prevent it from further happening in tertiary and primary hospitals for improvement of quality of life of the patient's.

\section{Study design}

It is a prospective, retrospective observational study conducted in a private and institutional hospitals of rural India.

\section{Methods}

A Prospective case study during daily ward rounds done on patients in rural hospital of India.

\section{Case History}

A 22-years-old male patient was admitted in private medical college hospital (S.V.S hospital, Mahabubnagar) with the complaint of fever for 15 days. The patient has been given aceclofenac, b-complex and T.cefixime orally twice a day. The drugs were started on $1^{\text {st }}$ Oct, 2015 and stopped on $7^{\text {th }}$ Oct, 2015. On $4^{\text {th }}$ Oct the patient developed erythromatous patches throughout the body (Steven-Johnsons Syndrome) particularly muco-cutaneous junctions. The laboratory data show serum creatinine $1.8 \mathrm{gm} / \mathrm{dl}$, blood urea $73 \mathrm{mg} / \mathrm{dl}, \mathrm{Hb} 15$ $\mathrm{g} / \mathrm{dl}$, TLC 6600 , neutrophils $60 \%$, eosinophils $2 \%$, lymphocytes $36 \%$, monocytes $2 \%$, platelets 1.9 lakhs and the tests for malarial and dengue parasites were negative. The drug was withdrawn after the advent of the reaction and the patient was given steroid, antibiotics like amoxicillin, metronidazole and symptomatic treatment (paracetamol, IV fluids). The patient was improved markedly and urea level was 
Citation: Chandaluri P, Prabhanjan M (2018) Steven Johnson Syndrome: Adverse Drug Reaction. J Gen Pract (Los Angel) 6: 349. doi:

Page 2 of 2

reverted back to normal. The reaction has never taken place after treatment.

\section{Discussion}

The SJS is usually caused by disorder of the immune system. The immune reaction can be triggered by infections, drugs or medications. As the patient doesn't have any kind of infections so the drugs prescribed are reviewed and stopped.

Cefixime has sulphur in its structure. The term sulphur allergy is misleading and dangerous and not be used. Patients with sulfonamide induced toxic epidermal necrolysis (TEN) have been shown to have a slow acetylator genotype resulting in increased production of sulfonamide hydroxylamine via $\mathrm{p}-450$ pathway. These drug metabolites have direct toxic effects or act via a hapten-mediated mechanism to break self-tolerance to endogenous proteins. This is the reason behind developing the erythematous patches due to the administration of cefixime, NSAIDS are also prescribed so the effect may be increased due to the co-administration of both the SJS causing drugs.

\section{Conclusion}

As aceclofenac and cefixime are frequently used concurrently, it is important to recognize their possible interaction. Early recognition of the ADR and removal of the offending drug can save the patient from unnecessary investigations and also reduce the medical expenses. The above ADR was discussed in the clinical review meeting, documented and submitted to the pharmacovigilance department in the hospital.

\section{Author's Statement}

The study conducted post approval from IRB/IEC from rural hospitals. This study has a limitation included lack of funding from organizations due to which it was not continued for a limited period of time. With the available funding a broader aspects in rural hospital organization and the methods to improve proactive role of clinical pharmacist could be implemented to minimize the risk of AE's and to improve the quality of life of the patient's.

\section{References}

1. Sundaram H, Huang PH, Hsu NJ, Huh CH, Wu WT, et al. (2016) Aesthetic applications of botulinum toxin A in Asians: An international, multidisciplinary, pan-asian consensus. Plast Reconstr Surg Glob Open 4: e872.

2. de Maio M, Swift A, Signorini M, Fagien S (2017) Facial assessment and injection guide for botulinum toxin and injectable hyaluronic acid fillers: Focus on the upper face. Plast Reconstr Surg 140: 265e.

3. Downie JB (2016) Global updates on the future directions of neurotoxins and fillers. Plast Reconstr Surg Glob Open 4: e1177. 